\title{
TEMPO-mediated oxidation of Flammulina velutipes mycelia for fabrication of transparent tension chitin membranes
}

\author{
Han Zhang ${ }^{1, a}$, Hao Liu ${ }^{1,2, b}$, Xiaofang Wan ${ }^{1,2, c}$, Jianhua Cheng ${ }^{2, d}$ \\ ${ }^{1}$ State Key Laboratory of Pulp \& Paper Engineering, South China University of Technology, \\ Guangzhou, Guangdong 510640, China \\ ${ }^{2}$ South China Institute of Collaborative Innovation, Dongguan, Guangdong 523808, China \\ azzzhhan@163.com, ${ }^{\text {b }}$ feliuh@scut.edu.cn, cwanxf@scut.edu.cn (Correspondence), \\ dchengjianhua@hrbeu.edu.cn
}

Keywords: 2,2,6,6-tetramethylpiperidine-1-oxyl (TEMPO), Flammulina velutipes mycelia (FvM), nanofiber, transparent, tension membrane.

Abstract. Mushroom mycelia have been investigated as a new source of chitin for fabrication of nano materials. This work demonstrated the importance of 2,2,6,6-tetramethylpiperidine-1-oxyl (TEMPO)-mediated oxidation in developing the functional qualities of chitin nanofibers using Flammulina velutipes mycelia (FvM) as the starting feedstock. Morphological characterization showed that the TEMPO treatment facilitated the formation of FvM membranes. The oxidation broke down the intact cellular structures. Homogeneous nanofibers were generated after the subsequent homogenization. The chemical analysis showed the ratio of $\mathrm{N}$ in FvM rather than $\mathrm{O}$ was increased by TEMPO oxidation. The carboxyl groups were not significantly introduced probably due to the degradation and dissolving of glucan, leaving chitin enriched in solids. TEMPO treatment also improved the thermal stability of FvM to a comparable level of Polyvinyl alcohol and Polyvinyl chloride. The membranes fabricated by crosslinking FvM nanofibers had excellent properties including high transparency, anti-UV activity and strong physical strength. The tensile index and elastic modulus were 2- and 2.5- folds of the reference polyethylene film. Our findings showed the TEMPO-oxidized FvM could be a promising material for fresh food packaging.

\section{Introduction}

There is a huge requirement for the natural biopolymers to meet the demand of packaging materials as a substitute of plastics. Chitin, the second most abundant polysaccharides, has shown great potential in the fabrication of biodegradable transparent membranes[1]. Generally, chitin has rigid crystalline structures and is insoluble in any routine solvent. It is derived from two major waste sources: shell of crustaceans and stalks of mushrooms [2]. Due to the poor manufacturing properties, it is more often to use chitin derivatives such as chitosan, carboxymethylchitosan, and nanofibers [3]. For example, chitin nanofibers from crabs and prawn shell have been obtained by Mushi et al.[4], showing good mechanical strength and thermal stability. However, the chitin in these shells has already been heavily calcified, mixed with a large portion of residual proteins. It requires mechanical grinding and removal of mineral and protein contaminants before use. The availability of these shells is quite seasonal and regional limited. It has been well known that mushroom cell walls have high content of chitin (30\% 50\%) that can be deacetylated to chitosan [2]. Many basidiomycete species are potential chitin sources, including Agaricus sp., Pleurotus sp., Ganoderma sp. and Lentinula sp. Since millions of tons of edible mushrooms are cultivated in China, the feedstocks are available both in quality and quantity. Although the nanofibers from mushroom chitin has been previously reported [3], the properties are still not satisfied, requiring further development through derivatization by selective oxidants such as 2,2,6,6-tetramethylpiperidine-1-oxyl (TEMPO). To the best knowledge of us, the effects of TEMPO on the morphologies, chemical structures and properties of mushroom mycelia have not been studied. In this work, we designed and investigated a new technical line to produce nanofibers and transparent tension membranes from residual Flammulina velutipes mycelia (See Fig. 1). Our findings may provide 
a promising approach for the manufacture of value-added sustainable materials from agricultural wastes.

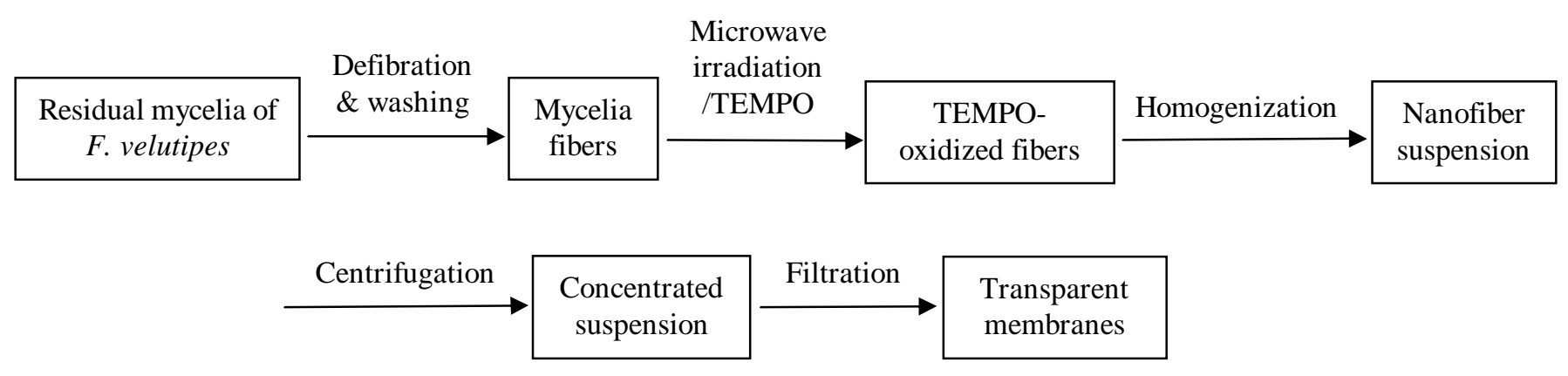

Fig. 1 Flow chart of the fabrication process

\section{Experimental}

Chemicals. 2,2,6,6-tetramethylpiperidine-1-oxyl (TEMPO), $\mathrm{NaClO}, \mathrm{NaClO}_{2}$ were purchased from Aladdin Chemical Co., Ltd. (Guangzhou, China). Other chemicals were supplied by Shanghai reagent company (Shanghai, China). All chemicals were of analytical grade and used without further purification.

Materials. Flammulina velutipes mycelia were collected from the residual fruit body after harvesting of mushroom. Fresh mycelia were defibrated in a defibrizer (Lorentzen \& Wettre, US) to generate individual fibers. After that, all the fibers were soaked in dilute $\mathrm{NaOH}$ solution $(5 \mathrm{~g} / \mathrm{L})$ to remove proteins, lipids and nucleic acids. Then the solids were rinsed with water and freeze-dried, marked as FvM.

TEMPO-mediated oxidation. FvM (10 g) was suspended in phosphate buffer ( $\mathrm{pH}$ 5.6), and stirred with addition of TEMPO $\left(0.01 \mathrm{~g} / \mathrm{g}_{\text {Biomass }}\right.$, on basis of dry weight $), \mathrm{NaClO}\left(5.0 \mathrm{~mL} / \mathrm{g}_{\text {Biomass }}\right), \mathrm{NaClO}_{2}(1.0$ $\left.\mathrm{g} / \mathrm{g}_{\text {Biomass }}\right)$. The mixture was then heated to $60 \square$ in a microwave oven $(500 \mathrm{~W})$ and maintained for $1 \mathrm{~h}$. Finally, the mixture was washed with deionized water to neutrality. The carboxyl content was determined as $0.15 \mathrm{mmol} / \mathrm{g}_{\text {Biomass }}$ using the conductometric titration method.

Homogenization. The dried oxidized FvM was dispersed in deionized water at a concentration of 0.5 $\%(\mathrm{w} / \mathrm{w})$ and then pumped into a high-pressure homogenization processor (Noozle, Mini, US). The operating pressure was set to be 1800 bar and the process was cycled for 4 times. The obtained suspension was centrifuged to remove redundant water.

Fabrication of transparent thin membranes. Homogenized samples were mixed with glycerol (10 $\mathrm{wt} \%)$ and filtered against a glass screen $(0.22 \mu \mathrm{m})$ under vacuum conditions. The FvM membrane was dried at $40 \square$ under temperature $23 \pm 1 \square$, relative humidity $50 \pm 2 \%$ for at least $48 \mathrm{~h}$ to achieve moisture balanced.

Characterization. Scanning electron microscopy (SEM) was performed at $23 \square$ on a SIGMA 300 field emission scanning electron microscopy (FESEM, ZEISS Merlin, Germany). Atomic force microscopy (AFM) was carried out in a Multimode Nanoscope IIIa system (Bruker, Germany). Fourier transform infrared spectroscopy (FTIR) spectra were recorded at room temperature on a Bruker Vertex 70v spectrometer. $3 \mathrm{mg}$ freeze-dried powders (raw mycelium and oxidized nanofibers) were diluted with $\mathrm{KBr}(300 \mathrm{mg})$ and scanned within a wave number range of $4000-400 \mathrm{~cm}^{-1}$. Thermal analysis (TG/DTA) was performed using a NETZSCH STA 449F3 thermal analyzer. To achieve this, 2-4 $\mathrm{mg}$ of the sample was heated from 30 to $700{ }^{\circ} \mathrm{C}$ at a rate of $10{ }^{\circ} \mathrm{C} / \mathrm{min}$ with $\mathrm{N}_{2}$ flow. Tensile tests were performed using a universal tensile tester (INSTRON 5565, China) following with the GB/T 1040.3-2006 Standard. The rectangular membranes (0.1 mm thickness and $15 \mathrm{~mm}$ width) and were tested at a strain rate of $1 \mathrm{~mm} / \mathrm{min}$. The Young's modulus was calculated from the initial linear region of the stress-strain curves. 


\section{Results and discussion}

Morphological characterization. Individual FvM fibers were successfully generated from the residual fruit body in mushroom cultures as shown in Figs. 2a\&b. SEM characterization also showed the fibers were smooth and intact with a diameter of about $20 \mu \mathrm{m}$. On the surface there are numerous micro-scaled fibrils randomly crosslinked with each other. Treatment with TEMPO broke down the intact cellular structure of FvM, peeled the cell wall into multiple layers and exposed the micro sheets and fibrils (Fig.2c). Obviously, the oxidant was capable of penetrating into the FvM fibers which allowed uniform reaction in the cell wall sublayers. Subsequent mechanical homogenization of FvM suspension resulted in transparent sol, consisting of uniform nanofibers with sizes of less than $100 \mathrm{~nm}$ in width and $1-5 \mu \mathrm{m}$ in length (Fig.2d). These nanofibers could crosslinked to form a smooth, transparent membrane (Fig.2e\&f).

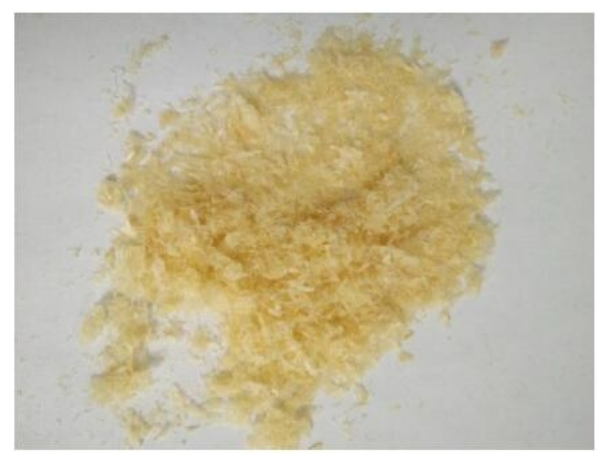

(a) Photo graph of mycelia

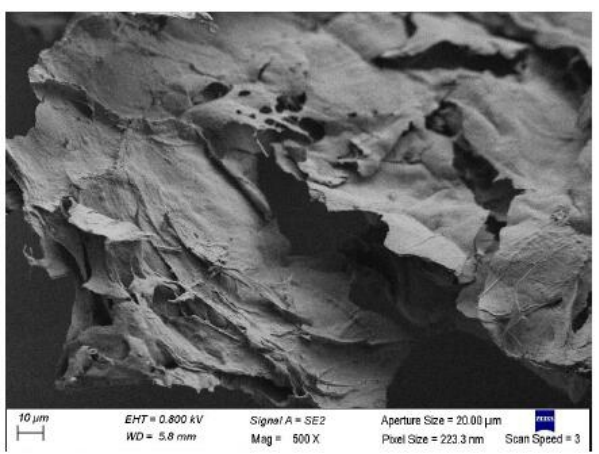

(c) SEM of TEMPO-oxidized mycelia

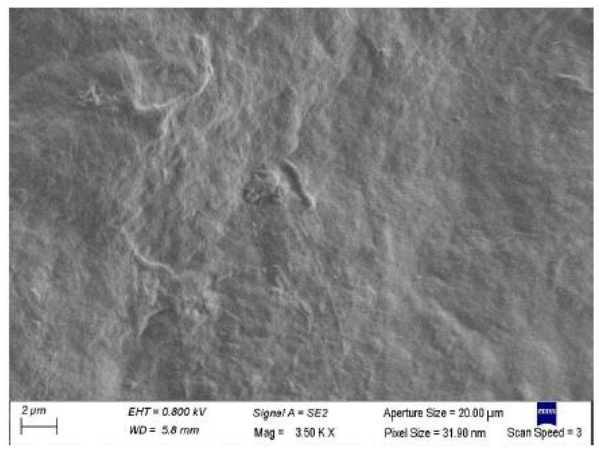

(e) SEM of transparent membrane

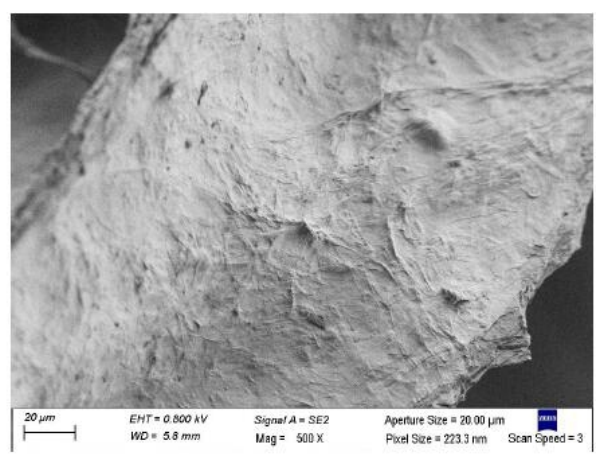

(b) SEM of mycelia

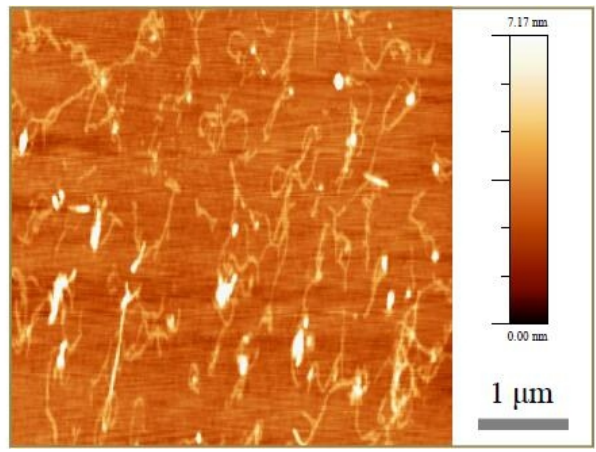

(d) AFM of nanofibers

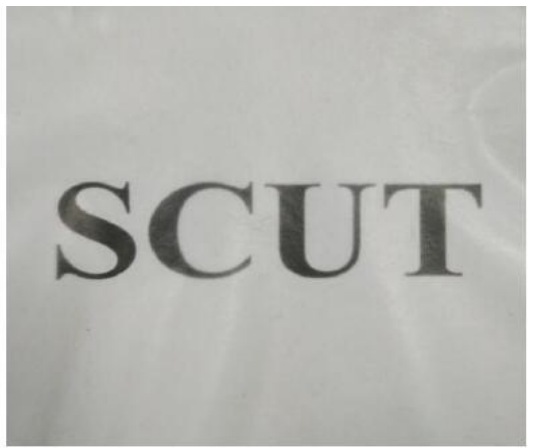

(f) Photo graph of transparent membrane

Fig. 2 Morphology of mycelia, nanofibers and membranes

Chemical characterization. Elemental compositions of FvM samples were summarized in Table 1. Clearly, the ratios of $\mathrm{N}, \mathrm{C}$ and $\mathrm{H}$ were increased after TEMPO treatment which is probably due to the chemical degradation of glucan in FvM. Particular, the significant increase in N content, i.e. by 32.64\%, 
suggested the TEMPO radicals did not eliminate the $\mathrm{N}$-acetyl-D-glucosamine groups. The ratio of $\mathrm{O}$ was decreased from $47.35 \%$ to $49.33 \%$, indicating the generation of carboxyl groups in glucose units led to dissolution of glucan. It has been reported that TEMPO selectively oxidized the primary hydroxyl group at the $\mathrm{C}_{6}$ site for cellulose, chitosan and many other dextrans[5,6]. Degradation and dissolution generally occurred in this reaction especially for those with lower molecular weight and crystallinity. Elimination of $\mathrm{S}$ suggested the complete removal of sulfur-containing protein contaminants.

Table 1 The elemental analysis of original and TEMPO-oxidized FvM

\begin{tabular}{cccccc}
\hline Samples & $\mathrm{C} / \%$ & $\mathrm{~N} / \%$ & $\mathrm{H} / \%$ & $\mathrm{O} / \%$ & $\mathrm{~S} / \%$ \\
\hline FvM & 41.39 & 2.88 & 6.41 & 49.33 & 0.02 \\
$\begin{array}{c}\text { TEMPO-oxidize } \\
\text { d FvM }\end{array}$ & 42.26 & 3.82 & 6.57 & 47.35 & 0 \\
\hline
\end{tabular}

FT-IR spectra (Fig.3) showed FvM and TEMPO-oxidized FvM shared similar bands at 3700-3000 $\mathrm{cm}^{-1}$ (-OH and $-\mathrm{NH}$ stretching vibration), 2924 and $2893 \mathrm{~cm}^{-1}$ (symmetric and asymmetric $-\mathrm{CH}$ stretching), $1415 \mathrm{~cm}^{-1}$ (-OH bending), and 1200-800 $\mathrm{cm}^{-1}$ (C-O and C-C stretching vibrations)[7]. Specifically, there was a sharp peak at $1561 \mathrm{~cm}^{-1}$ which was attributed to the $\mathrm{C}-\mathrm{N}$ groups in the chitin. This peak red-shifted to $1552 \mathrm{~cm}^{-1}$ after oxidation with TEMPO radicals, corresponding to slight increase in the polarity of materials. However, no significant signal change at the carboxyl site $(\sim 1720$ $\mathrm{cm}^{-1}$ ) was found in Fig. 3. This confirmed that TEMPO-mediated oxidation did not increase the number of carboxyl groups in chitin. Instead, those amorphous glucan moieties were more readily attacked and dissolved.

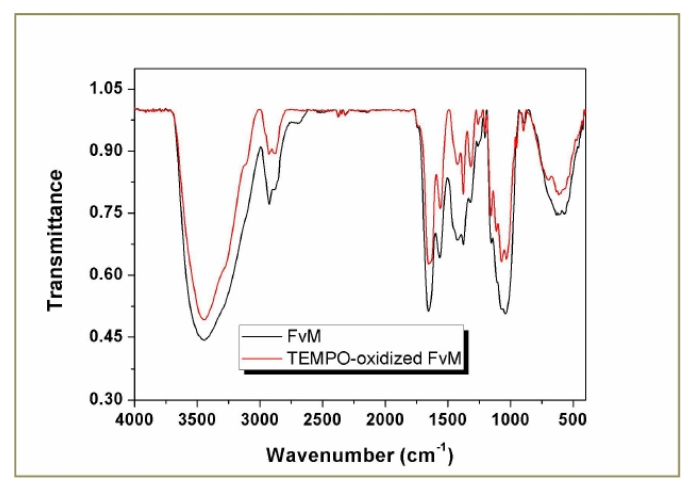

Fig.3 FT-IR spectra of FvM

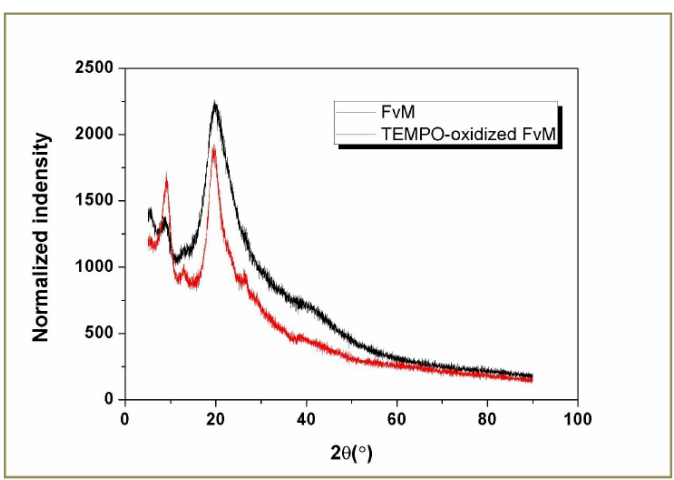

Fig.4 XRD spectra of FvM and TEMPO-oxidized FvM

XRD spectra of FvM samples were illustrated in Fig.4. There are two main diffraction peaks at 9.11 and $19.57^{\circ}$ which correspond to the 020 and 110 planes, respectively. They are typical crystal patterns of $\alpha$-chitin[8]. Thereafter, TEMPO-mediated oxidization did not destroy the crystal structure of chitin in the material. More intensive crystal structure was observed probably due to the loss of partly-dissolved glucans.

Transparency and UV absorbability. UV/visible spectra of FvM membrane were obtained by attaching the sample on the outer surface of a cuvette cell $(1 \mathrm{~cm}$, full of water). PE films with the comparable thickness were used as reference. The results in Fig. 5 showed the FvM membrane had much higher absorbance at both UV and visible regions. The light transmittance at $460 \mathrm{~nm}$ was much lower than the PE film although it also appeared transparent when attached close to an objective (Fig.2f). The strong absorbance at UV region (200 340) showed our material had excellent anti-UV 
properties over the PE film. Such features impart the FvM membrane potential use as an anti-browning package material for fresh vegetables such as mushrooms.

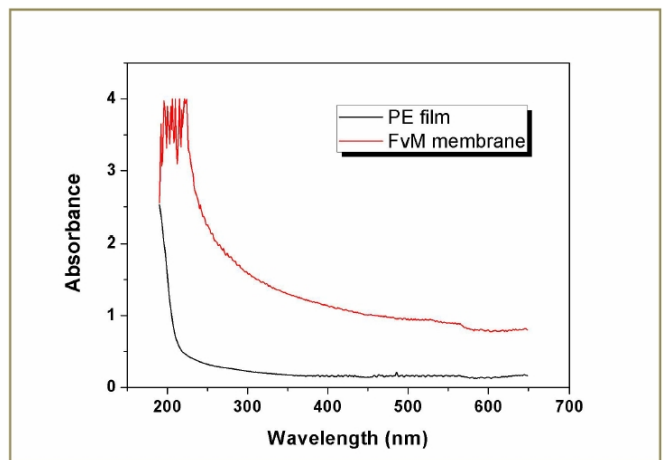

Fig.5 UV/visible spectra of PE film and FvM Membrane

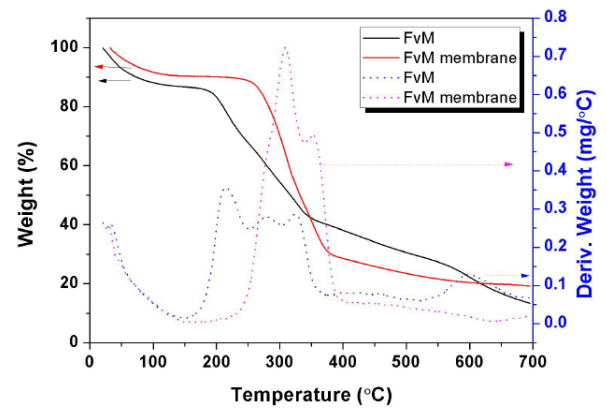

Fig. 6 Thermal stability of FvM and TEMPO-oxidized FvM

Note: $42 \pm 0.5 \mu \mathrm{m}$ in thickness

Tensile strength. FvM membranes had excellent physical strength from the tension test as list in Table 2. The tensile strength and elastic modulus were 2- and 2.5- folds of the reference PE film. This indicates the molecules of TEMPO-oxidized chitin/glucan in FvM could readily form intermolecular hydrogen bonds. However, the elongation at breaking was much lower for FvM membrane. This suggested that the new material was less flexible and easily broken. Such properties could be improved by blending with a small amount of PVC, i.e. $1 \%$.

Thermal stability. TG and TGA tests proved that the TEMPO treatment promoted the stability of FvM fibers. The critical point for thermal degradation was prolonged from 175 to $250^{\circ} \mathrm{C}$, near the points of PVA and PVC plastics $[9,10]$. An explanation was that TEMPO degraded and removed those low-molecular-weight glucan content which was usually unstable in heating conditions. Customers generally require the transparent film could tolerate regular heating conditions such as microwave heating. A higher thermal stability is important for maintaining the intact coverage and minimizing the generation of volatile organic compounds.

Table 2 Mechanical properties of FvM membrane and PE film

\begin{tabular}{cccc}
\hline Sample type & $\begin{array}{c}\text { Ultimate tensile } \\
\text { strength }(\mathrm{MPa})\end{array}$ & Elastic modulus (MPa) & $\begin{array}{c}\text { Elongation at } \\
\text { breaking/\% }\end{array}$ \\
\hline FvM membrane & 23.6 & 2020 & 2 \\
PE film & 11.0 & 84 & 458 \\
\hline
\end{tabular}

\section{Conclusions}

TEMPO-mediated oxidation broke down the intact cellular structure of Flammulina velutipes mycelia (FvM) and facilitated the generation of homogeneous nanofibers. TEMPO treatment increased the content of $\mathrm{N}$ but decreased $\mathrm{O}$ in FvM. Moreover, the carboxyl groups were not significantly introduced. Therefore, TEMPO probably degraded and dissolved glucan, leaving the N-acetyl-D-glucosamine groups in chitin less destroyed. This led to the improvement in the thermal stability. The FvM nanofibers could crosslinked to form transparent tension membranes with excellent anti-UV properties. The tensile strength and elastic modulus were 2- and 2.5- folds of the reference PE film. Our findings showed the TEMPO-oxidized FvM could be a promising material for fresh food packaging. 


\section{Acknowledgements}

This work was financially supported by the Foundation of State Key Laboratory of Pulp and Paper Engineering ( No.2017PY01 \& No.2017QN01), the Science and Technology Program of Guangzhou (No.201704020038 \& No.201607020025), the Guangdong Natural Science Foundation (No.2017A030310341) and the Fundamental Research Funds for the Central Universities (No.x2qsD2142050).

\section{References}

[1] S. K. Biswas, M. I. Shams, A. K. Das, M. N. Islam and M. M. Nazhad: Fiber. Polym. Vol. 16(4) (2015), p. 774-781.

[2] T. Wu, S. Zivanovic, F. A. Draughon and C. E. Sams: J. Agr. Food Chem. Vol. 52(26) (2004), p. 7905-7910.

[3] S. Ifuku, M. Nogi, K. Abe, M. Yoshioka, M. Morimoto, H. Saimoto and H. Yano: Carbohyd. Polym. Vol. 84(2) (2011), p. 762-764.

[4] N. E. Mushi, N. Butchosa, M. Salajkova, Q. Zhou and L. A. Berglund: Carbohyd. Polym. Vol. 112 (2014), p. 255-263.

[5] I. Shibata and A. Isogai: Cellulose Vol. 10(2) (2003), p. 151-158.

[6] Y. Okita, T. Saito and A. Isogai: Biomacromolecules Vol. 11(6) (2010), p. 1696-1700.

[7] Y. Fan, T. Saito and A. Isogai: Biomacromolecules Vol. 9(1) (2007), p. 192-198.

[8] G. Cárdenas, G. Cabrera, E. Taboada and S. P. Miranda: J. Appl. Polym. Sci. Vol. 93(4) (2004), p. 1876-1885.

[9] B. Shen, W. Zhai, D. Lu, J. Wang and W. Zheng: RSC Adv. Vol. 2(11) (2012), p. 4713-4719.

[10] M. Hasan, R. Kumar, M. A. Barakat and M. Lee: RSC Adv. Vol. 5(19) (2015), p. 14393-14399. 